\title{
LIMITS FOR THE CHARACTERISTIC ROOTS OF A MATRIX ${ }^{1}$
}

\section{A. B. FARNELL}

Let $A$ be a square matrix of order $n$ with complex numbers as elements. The equation $|\lambda I-A|=0$ is called the characteristic equation of the matrix $A$, and the roots $\lambda_{i}$, the characteristic roots of the matrix $A$. Although it is not possible to make any definite statements regarding the nature of the characteristic roots for the general matrix, several authors have given upper limits to the roots. In 1900, Bendixson $[1]^{2}$ obtained upper limits for the real and imaginary parts of the characteristic roots of a real matrix. In 1902, Hirsch [5] extended these results to matrices with complex numbers as elements. A limit was also given by Bromwich [2] in 1904. These limits were further refined by Browne [3] in 1930, and by Parker [7] in 1937.

In 1918, Toeplitz [8], using the results of Bendixson and Hirsch, studied the algebraic form $(A x \mid x) \equiv \sum_{r, 8} a_{r s} x_{s} \bar{x}_{r}$ corresponding to the matrix $A$, where by hypothesis the algebraic form $(x \mid x)=\sum_{r=1}^{n} x_{r} \bar{x}_{r}$ has the value unity. By using the fact that $A$ may be decomposed uniquely in the form $A=B+i C$, where $B$ and $C$ are Hermitian, he showed that the totality of values which the algebraic form assumes lie within a rectangle with sides parallel to the real and imaginary axes. He further showed that this field of values is bounded by a convex curve. Hausdorff [4] showed that the field of values is connected, bounded, closed, and convex. In 1932, Murnaghan [6], using the fact that $(A x \mid x)$ has values invariant under unitary transformations of $A$, showed that for normal matrices (that is, matrices which can be transformed unitarily into diagonal form) the field of values is a convex polygon. For non-normal matrices, he showed that in the general case the characteristic roots of the matrix are the foci of the curve bounding the field of values. Wintner had previously remarked that for $n=2$ the curve is an ellipse. The first three theorems below give radii of circles within which not merely the characteristic roots but also the entire field of values lies.

Let $A^{\prime}$ and $\bar{A}$ denote the transpose and conjugate, respectively, of the matrix $A$, and write

$$
B=\left(A+\bar{A}^{\prime}\right) / 2, \quad C=\left(A-\bar{A}^{\prime}\right) / 2 i .
$$

Received by the editors March 10, 1944, and, in revised form, April 10, 1944.

1 Partially extracted from a dissertation, University of California, 1944.

2 Numbers in brackets refer to the Bibliography at the end of the paper. 
It is evident that $B$ and $C$ are Hermitian. The theorem given by Parker may be stated as follows:

PARKer's Theorem. If $A$ is any square matrix, and if $2 S r, 2 S^{\prime} r$, $2 S^{\prime \prime} r$ are the sums of the absolute values of the elements in the rth row and the absolute values of the elements in the rth column of $A, B$, and $C$, respectively, and if $S, S^{\prime}, S^{\prime \prime}$ are the greatest of the $S r, S^{\prime} r, S^{\prime \prime} r$, respectively, then for any characteristic root, $\lambda=\alpha+i \beta$, of $A$, we have

$$
|\lambda| \leqq S, \quad|\alpha| \leqq S^{\prime}, \quad|\beta| \leqq S^{\prime \prime} .
$$

If $\lambda=\alpha+i \beta$ is a characteristic root of a matrix $A=\left(a_{r s}\right)$ of order $n$, there exists a set of numbers $\left(x_{1}, x_{2}, \cdots, x_{n}\right)$ such that

$$
\sum_{r=1}^{n} x_{r} \bar{x}_{r}=1
$$

which satisfy the relations

$$
\lambda x_{r}=\sum_{s} a_{r s} x_{s} \quad(r=1,2, \cdots, n) .
$$

If we multiply the $r$ th equation in (1) by $\bar{x}_{r}$, and sum as to $r$, we obtain

$$
\lambda=\sum_{r, s} a_{r s} \bar{x}_{r} x_{s} .
$$

The numbers $\sum a_{r s} \bar{x}_{r} x_{s}$, where $\sum x_{r} \bar{x}_{r}$ has the value unity, comprise the field of values of $A$. Hence (2) shows that the characteristic roots lie within the field of values. In the following, $\lambda$ will be considered to be any value in this field, the conclusions holding a fortiori where $\lambda$ is a characteristic root.

Taking conjugates of both sides of (2), and interchanging subscripts, we have

$$
\bar{\lambda}=\sum_{r, s} \bar{a}_{s r} \bar{x}_{r} x_{s} .
$$

From (2) and (3), by addition and subtraction, it follows that

$$
\begin{aligned}
\alpha & =\sum_{r, s} b_{r s} \bar{x}_{r} x_{s}, \\
\beta & =\sum_{r, s} c_{r s} \bar{x}_{r} x_{s} .
\end{aligned}
$$

From the relations (2) and (4), upper limits for $|\lambda|,|\alpha|$, and $|\beta|$ may be obtained. Since these relations are identical in form, it is suffcient to carry through the computations for one of them. Further- 
more, the theorems will be stated briefly since the context will be evident.

Several results will now be established. The best upper limit may be given by one in some cases, by another in others.

THEOREM 1. Let $R_{r}=\sum_{s}\left|a_{r s}\right|, \quad T_{s}=\sum_{r}\left|a_{r s}\right|, \quad R=\max \left(R_{r}\right)$, $T=\max \left(T_{s}\right)$. Then

$$
|\lambda| \leqq(R T)^{1 / 2}
$$

Let $\xi_{r}=\left|x_{r}\right|$, so that

$$
\sum_{r} \xi_{r}^{2}=1
$$

If we take the absolute value of (2), we have

$$
|\lambda| \leqq \sum_{r, s}\left|a_{r s}\right| \xi_{r} \xi_{s}
$$

Rewriting $\left|a_{r s}\right| \xi_{r} \xi_{s}$ as $\left|a_{r s}\right|^{1 / 2} \xi_{r} \cdot\left|a_{r s}\right|^{1 / 2} \xi_{s}$, and applying Schwarz's inequality, it follows that

$$
\begin{aligned}
|\lambda| & \leqq\left(\sum_{r, s}\left|a_{r s}\right| \xi_{r}^{2}\right)^{1 / 2}\left(\sum_{r, s}\left|a_{r s}\right| \xi_{s}^{2}\right)^{1 / 2} \\
& =\left(\sum_{r} R_{r} \xi_{r}^{2}\right)^{1 / 2}\left(\sum_{s} T_{s} \xi_{s}^{2}\right)^{1 / 2} \\
& \leqq\left(R \sum_{r} \xi_{r}^{2}\right)^{1 / 2}\left(T \sum_{s} \xi_{s}^{2}\right)^{1 / 2} \\
& =(R T)^{1 / 2} .
\end{aligned}
$$

Theorem 2. If $\lambda$ lies in the field of values of $A$, then,

$$
|\lambda| \leqq\left(\sum_{r, s}\left|a_{r s}\right|^{2}\right)^{1 / 2}
$$

Rewriting $\left|a_{r s}\right| \xi_{r} \xi_{s}$ in (5) as $\left|a_{r s}\right| \cdot \xi_{r} \xi_{s}$, and applying Schwarz's inequality, we have

$$
\begin{aligned}
|\lambda| & \leqq\left(\sum_{r, s}\left|a_{r s}\right|^{2}\right)^{1 / 2}\left(\sum_{r, 8} \xi_{r}^{2} \xi_{s}^{2}\right)^{1 / 2} \\
& =\left(\sum_{r, s}\left|a_{r s}\right|^{2}\right)^{1 / 2}\left(\left(\sum_{r} \xi_{r}^{2}\right)^{2}\right)^{1 / 2} \\
& =\left(\sum_{r, s}\left|a_{r s}\right|^{2}\right)^{1 / 2} .
\end{aligned}
$$


THEOREM 3. If $\lambda$ lies in the field of values of $A$, then $|\lambda| \leqq M$, where $M$ is defined by (7) below.

From (2),

$$
\begin{aligned}
|\lambda|= & \left|\sum_{r, s} a_{r s} \bar{x}_{r} x_{s}\right| \\
\leqq & \frac{1}{n} \sum_{r}\left|a_{r r}\right|+\frac{1}{n} \sum_{s} \sum_{r<s}\left(\left|a_{r r}\right|-\left|a_{s s}\right|\right)\left(\xi_{r}^{2}-\xi_{s}^{2}\right) \\
& +2 \sum_{s} \sum_{r<s}\left|a_{r s}\right| \xi_{r} \xi_{s} \\
= & \frac{1}{n} \sum_{r}\left|a_{r r}\right|+\sum_{s} \sum_{r<s} \frac{(n-1)^{1 / 2}}{n}\left(\left|a_{r r}\right|-\left|a_{s s}\right|\right) \cdot \frac{\xi_{r}^{2}-\xi_{s}^{2}}{(n-1)^{1 / 2}} \\
& +2 \sum_{s} \sum_{r<s}\left(\frac{n-1}{2 n}\right)^{1 / 2}\left|a_{r s}\right| \cdot\left(\frac{2 n}{n-1}\right)^{1 / 2} \xi_{r} \xi_{s} \\
\leqq & \frac{1}{n} \sum_{r}\left|a_{r r}\right|+\left\{\frac{n-1}{n^{2}} \sum_{s} \sum_{r<s}\left(\left|a_{r r}\right|-\left|a_{s s}\right|\right)^{2}\right. \\
& \left.+\frac{n-1}{2 n} \sum_{s} \sum_{r<s}\left(\left|a_{r s}\right|+\left|a_{s r}\right|\right)^{2}\right\}^{1 / 2}
\end{aligned}
$$

by Schwarz's inequality, and the fact that $\sum_{r} \xi_{r}^{2}=1$. That is, $|\lambda| \leqq M$, where

$$
\begin{gathered}
M=\frac{1}{n}\left[\sum_{r}\left|a_{r r}\right|+\left\{(n-1) \sum_{s} \sum_{r<s}\left(\left|a_{r r}\right|-\left|a_{s s}\right|\right)^{2}\right.\right. \\
\left.\left.+\frac{n(n-1)}{2} \sum_{s} \sum_{r<s}\left(\left|a_{r s}\right|+\left|a_{s r}\right|\right)^{2}\right\}^{1 / 2}\right] .
\end{gathered}
$$

For $n=2, M$ is the maximum value of the right-hand side of (6), and hence the minimum for this type of upper limit. For $n>2$, the form of the maximum for the right-hand side of (6) is too complicated for practical use.

THEOREM 4. Let $U_{r}=\sum_{s}\left|a_{r s}\right|^{2}, \quad V_{s}=\sum_{r}\left|a_{r s}\right|^{2}$. Then $|\lambda|$ $\leqq\left(\sum_{r}\left(U_{r} V_{r}\right)^{1 / 2}\right)^{1 / 2}$.

From (1),

$$
\lambda x_{r}=\sum_{s} a_{r s} x_{s}
$$

Multiplying each by $\lambda$, and replacing $\lambda x_{r}$ from (1), we have 


$$
\begin{aligned}
& \lambda^{2} x_{1}=a_{11}\left(a_{11} x_{1}+a_{12} x_{2}+\cdots+a_{1 n} x_{n}\right)+\cdots \\
& +a_{1 n}\left(a_{n 1} x_{1}+a_{n 2} x_{2}+\cdots+a_{n n} x_{n}\right) \text {, } \\
& \lambda^{2} x_{n}=a_{n 1}\left(a_{11} x_{1}+a_{12} x_{2}+\cdots+a_{1 n} x_{n}\right)+\cdots \\
& +a_{n n}\left(a_{n 1} x_{1}+a_{n 2} x_{2}+\cdots+a_{n n} x_{n}\right) .
\end{aligned}
$$

Multiplying the $r$ th equation by $\bar{x}_{r}$, adding the $n$ equations, and taking the absolute value of both sides, we get

$$
\begin{aligned}
\lambda^{2} \leqq & \left(\left|a_{11}\right| \xi_{1}+\left|a_{21}\right| \xi_{2}+\cdots+\left|a_{n 1}\right| \xi_{n}\right)\left(\left|a_{11}\right| \xi_{1}+\left|a_{12}\right| \xi_{2}\right. \\
& \left.+\cdots+\left|a_{1 n}\right| \xi_{n}\right)+\cdots+\left(\left|a_{1 n}\right| \xi_{1}+\left|a_{2 n}\right| \xi_{2}+\cdots\right. \\
& \left.+\left|a_{n n}\right| \xi_{n}\right)\left(\left|a_{n 1}\right| \xi_{1}+\left|a_{n 2}\right| \xi_{2}+\cdots+\left|a_{n n}\right| \xi_{n}\right) \\
\leqq & \sum_{r}\left(U_{r} V_{r}\right)^{1 / 2}
\end{aligned}
$$

since, by Schwarz's inequality, $\sum_{r} c_{r} \xi_{r} \leqq\left(\sum_{r} c_{r}^{2}\right)^{1 / 2}\left(\sum_{r} \xi_{r}^{2}\right)^{1 / 2}=\left(\sum_{r} c_{r}^{2}\right)^{1 / 2}$.

TheOREM 5. For $n=2,|\lambda| \leqq(R T)^{1 / 2}$, where $R T=\max \left(R_{r} T_{r}\right)$.

From (8),

$$
\begin{aligned}
\lambda^{2} \leqq & \left(\left|a_{11}\right| \xi_{1}+\left|a_{12}\right| \xi_{2}\right)\left(\left|a_{11}\right| \xi_{1}+\left|a_{21}\right| \xi_{2}\right) \\
& +\left(\left|a_{12}\right| \xi_{1}+\left|a_{22}\right| \xi_{2}\right)\left(\left|a_{21}\right| \xi_{1}+\left|a_{22}\right| \xi_{2}\right) .
\end{aligned}
$$

Here, for example, $R_{2} T_{2} \leqq R_{1} T_{1}$ implies $\left|a_{22}\right| \leqq\left|a_{11}\right|$. Then since $\xi_{1} \xi_{2} \leqq 1 / 2$, (9) gives,

$$
\lambda^{2} \leqq R_{1} T_{1}
$$

The probability of the truth of this theorem was suggested to me by W. V. Parker. Although no contradictions have been found, no method of proof has been discovered for $n>2$.

The effectiveness of the above theorems can sometimes be improved by using the fact that if each diagonal element of a matrix $A$ is increased by $a$, the numbers $\lambda$ comprising the field of values of the matrix will be increased by $a$.

Let $L$ denote the best upper limit obtained for the absolute value of $\lambda$.

THEOREM 6. Let a represent the absolute value of the determinant of $A$. Then

$$
a \leqq L^{n}
$$

This follows directly from the fact that $a=\left|\lambda_{1} \lambda_{2} \cdots \lambda_{n}\right|$.

It is interesting to note that this result is frequently better than 
the well known upper bound given by Hadamard: "If the elements of the matrix $\left(a_{r s}\right)$ with $r, s \leqq n$ satisfy the condition $\left|a_{r s}\right| \leqq B$, then the following inequality holds:

$$
\left|\operatorname{det}\left[a_{r 8}\right]\right| \leqq B^{n} n^{n / 2} \cdot "
$$

\section{BIBLIOGRAPHY}

1. Bendixson, Sur les racines d'une équation fondamentale, Acta Math. vol. 25 (1902) pp. 359-365.

2. Bromwich, On the roots of the characteristic equation of a linear substitution, Acta Math. vol. 30 (1906) pp. 295-304.

3. Browne, The characteristic roots of a matrix, Bull. Amer. Math. Soc. vol. 36 (1930) pp. 705-710. 316.

4. Hausdorff, Der Wertvorrat einer Bilinearform, Math. Zeit. vol. 3 (1919) pp. 314-

5. Hirsch, Sur les racines d'une équation fondamentale, Acta Math. vol. 25 (1902) pp. $367-370$.

6. Murnaghan, On the field of values of a square matrix, Proc. Nat. Acad. Sci. U.S.A. vol. 18 (1932) pp. 246-248. 487.

7. Parker, The characteristic roots of a matrix, Duke Math. J. vol. 3 (1937) pp. 484-

8. Toeplitz, Das algebraische Analogon zu einem Satze von Fejêr, Math. Zeit. vol. 2 (1918) pp. 187-197.

United States Military Academy 